\title{
Dor em doentes com lesões raquimedulares
}

\author{
Manoel Jacobsen Teixeira*, Lin Tchia Yeng**, Marcos Montagnini***, Romélio Cristian \\ Peña Calvimontes ${ }^{\star * * *}$
}

Clínica Neurológica do Hospital das Clínicas da FMUSP, São Paulo, SP

\section{RESUMO}

A dor crônica é um sintoma comum em doentes que sofrem lesões raquimedulares. Os autores apresentam os aspectos demográficos, as características clínicas e os resultados do tratamento de 155 doentes com dor causada por lesão raquimedular. A mediana das idades foi 47,1 anos, o período de duração da dor teve mediana de 61,7 meses. As causas mais comuns das lesões foram ferimentos por projéteis de arma de fogo $(30,3 \%)$, traumatismos fechados $(28,4 \%)$ e as iatrogenias $(18,7 \%)$. Os segmentos mais freqüentemente acometidos foram o torácico $(43,2 \%)$, seguindo-se a região lombar e a cervical. A dor localizou-se predominantemente nos membros inferiores $75,4 \%$. A dor fantasma foi observada em $88,3 \%$ dos doentes e a miofascial em $25 \%$. Os padrões mais comuns de dor foram o queimor (32,3\%) e o queimor e choque $(29,0 \%)$. O fator que mais condicionou a melhora da dor foi o repouso $(44,5 \%)$ e o mais relacionado à piora foram os exercícios $(65,9 \%)$ e os estresses psíquicos $(61,9 \%)$. Todos os doentes foram tratados com medicina física e com antidepressivos tricíclicos, fenotiazinas e analgésicos antiinflamatórios não-hormonais; cerca de 1/3 dos doentes foi medicado com diazepínicos, morfínicos, baclofeno, carbamazepina ou outros agentes. O tratamento farmacológico e fisiátrico proporcionou resultados satisfatórios em $62 \%$ dos doentes. A rizotomia percutânea por radiofreqüência, indicada em três casos, proporcionou melhora significativa em um; a estimulação elétrica epidural indicada em 42 doentes proporcionou melhora significante em 17; a lesão do trato de Lissauer e do corno posterior da medula espinal indicada para o tratamento de 21 doentes proporcionou melhora significante em 14; e a infusão de agentes morfínicos no compartimento epidural proporcionou melhora significante em apenas dois de seis doentes.

Concluiu-se que a dor em doentes com lesões raquimedulares é freqüentemente rebelde ao tratamento e que a seqüência de prescrição de analgésicos e psicotrópicos, medicina física e, quando necessário, medidas cirúrgicas, incluindo a estimulação elétrica epidural e a lesão do trato de Lissauer e do corno posterior da medula espinal proporcionam melhora satisfatória em significante número de casos.

\section{PALAVRAS-CHAVE}

Dor crônica. Mielopatia. Radiculopatia. Traumatismo raquimedular.

\section{ABSTRACT}

Pain in spine injuried patients

Chronic pain is frequent in patients presenting spinal cord and spinal roots lesions. The characteristics and the results of the treatment of 155 patients with spinal lesions are presented.

The median of the ages was 47.1 years, and the median duration of the pain complaints was 61.7 months. The most common aetiologies of the lesions were gunshot injuries of the spine (30.3\%), closed lesions $(28.4 \%)$ and iatrogenies (18.7\%). Thoracic spine was the most frequent segment involved, followed by lumbar and cervical spinal segments. Pain was more frequent in the lower limbs $75.4 \%$. Phantom pain was observed in $88.3 \%$ of the cases and myofascial pain in $25.0 \%$. Burning (32.3\%) and burning and stabbing

* Neurocirurgião, Professor Doutor do Departamento de Neurologia da Faculdade de Medicina da Universidade de São Paulo.

* Médica fisiatra da Divisão de Medicina Física do Instituto de Ortopedia e Traumatologia do Hospital das Clínicas da Faculdade de Medicina da Universidade de São Paulo.

*** Médico fisiatra, Assistant Professor do Medical College of Wisconsin, EUA

**** Médico observador do Centro de Dor do Hospital das Clínicas da Faculdade de Medicina da Universidade de São Paulo. 
pains $(29.0 \%)$ were the most common pattern of complaints. Pain was very often aggravated by exercices (65.9\%) and psychogenic factors (61.9\%), and was atenuated by resting in $44.5 \%$ of the cases. All patients were treated with physical medicine, and tryciclic antidepressants, phenotiazines and non steroidal antiinflammatory drugs; and 1/3 of them with diazepines, opiates, baclofen, carbamazepine and other agents. Pharmachological and physiatric treatment resulted in satisfactory improvement in $62 \%$ of the patients. Percutaneous spinal roots rhyzotomies were performed in three cases, and resulted in improvement in one. Spinal cord electric stimulation performed in 42 patients, resulted in significant improvement of pain in 17 cases; the DREZ, performed in 21 patients, resulted in improvement in 14 and the spinal epidural infusion of morphine resulted in improvement in just two of the six cases so treated.

It was concluded that spinal lesions pains are very often incapacitating and not easely controlled. The prescription of psychotropics, anticonvulsants, physical medicine, and when necessary functional neurosurgical procedures are recommended for its control.

\section{KEYWORDS}

Chronic pain. Myelopathy. Radiculopathy. Spinal cord lesions.

\section{Introdução}

A dor é uma complicação freqüente e séria nos doentes que apresentam lesão na medula espinal e da cauda eqüina ${ }^{44}$. Manifesta-se, cronicamente, em 6,3\% a 100\% dos casos e é causa de grave incapacidade em $27 \%$ a $46 \%$ daqueles que dela padecem ${ }^{35,54}$; cerca de $10 \%$ dos doentes com dor por lesão medular e de cauda eqüina necessitam de tratamento neurocirúrgico funcional antiálgico ${ }^{10,15,16,57}$. A dor é usualmente referida em regiões nas quais a sensibilidade está parcial ou totalmente comprometida. $\mathrm{O}$ escasso conhecimento sobre a fisiopatologia da dor neuropática é uma das razões pelas quais vários métodos foram propostos para o seu controle. O objetivo do presente trabalho é o de apresentar os resultados da avaliação clínica e de algumas modalidades de tratamento de um grupo de doentes com dor crônica resultante de lesões raquimedulares atendidos em uma unidade especializada de tratamento de dor de um hospital geral de um grande centro urbano no Brasil.

\section{Casuística e métodos}

Este trabalho é baseado na observação de 155 doentes com dor resultante de mielopatias ou de lesões da cauda eqüina avaliados na clínica neurológica do Hospital das Clínicas da Faculdade de Medicina da Universidade de São Paulo, entre junho de 1979 e março de 1990.
Eram do sexo masculino $65,2 \%$ dos doentes. As idades variaram de 16 a 82 anos (mediana $=47,1$ anos). O período de duração da síndrome álgica variou de 1 a 564 meses (mediana 61,7 meses).

\section{Resultados}

Ocorreu predomínio dos traumatismos raquimedulares por ferimentos por projéteis de arma de fogo $(30,3 \%)$, seguindo-se os traumatismos fechados $(28,4 \%)$, as lesões iatrogênicas e as lesões neoplásicas (Tabela 1).

\begin{tabular}{lcr}
\hline \multicolumn{3}{c}{ Tabela 1 } \\
Distribuição dos doentes segundo a etiologia da lesão \\
raquimedular
\end{tabular}

A lesão localizou-se predominantemente na região da medula espinal torácica (Tabela 2).

Tabela 2

Distribuição dos doentes segundo o segmento do canal raquidiano acometido

\begin{tabular}{lcc}
\hline Segmento & $\mathbf{n}$ & $\mathbf{\%}$ \\
\hline Torácico & 67 & 43,2 \\
Lombar & 53 & 34,2 \\
Cervical & 35 & 22,6 \\
\hline Total & $\mathbf{1 5 5}$ & $\mathbf{1 0 0}$ \\
\hline
\end{tabular}

A maioria dos doentes referiu sensação de dor em queimor ou queimor e choque (Tabela 3).

Tabela 3

Distribuição dos doentes quanto às qualidades das sensações dolorosas predominantes

\begin{tabular}{lrr}
\hline Padrão da dor & $\mathbf{n}$ & $\mathbf{\%}$ \\
\hline Queimor & 50 & 32,3 \\
Queimor/choque & 45 & 29,0 \\
Queimor/pressão & 14 & 9,0 \\
Choque & 11 & 7,1 \\
Peso & 8 & 5,2 \\
Queimor/formigamento & 6 & 3,9 \\
Outras & 21 & 13,5 \\
\hline Total & $\mathbf{1 5 5}$ & 100 \\
\hline
\end{tabular}


A maioria dos doentes apresentava dor nos membros inferiores (Tabela 4).

\begin{tabular}{|c|c|c|}
\hline \multicolumn{3}{|c|}{$\begin{array}{c}\text { Tabela } 4 \\
\text { Distribuição dos doentes segundo a localização da dor }\end{array}$} \\
\hline Localização da dor & $\mathbf{n}$ & $\%$ \\
\hline Membros inferiores & 84 & 54,1 \\
\hline Tronco e membros inferiores & 33 & 21,3 \\
\hline Tronco & 19 & 12,3 \\
\hline Outras & 21 & 13,5 \\
\hline
\end{tabular}

Havia dor fantasma aos segmentos desaferentados em $88,3 \%$ dos casos, dor miofascial em $25 \%$ e dor segmentar em 15,4\%. Em 4,1\% dos doentes, o padrão da dor não foi caracterizado ou a dor foi visceral (Tabela 5).

\begin{tabular}{lcr}
\hline \multicolumn{4}{c}{ Tabela } & 5 \\
\multicolumn{1}{c}{ Distribuição dos doentes } & segundo o padrão da dor \\
\hline Padrões de dor & $\mathbf{n}$ & $\%$ \\
\hline Fantasma & 137 & 88,3 \\
Miofascial & 18 & 25,0 \\
Segmentar & 24 & 15,4 \\
Outros & 6 & 4,1 \\
\hline
\end{tabular}

Havia relato de melhora da dor com o repouso em $44,5 \%$ dos casos, com a postura em $14,8 \%$, com os exercícios em $12,9 \%$ e com massageamento em 8,4\%; não ocorria melhora com manobras em 15,5\% dos doentes (Tabela 6).

\begin{tabular}{|c|c|c|}
\hline \multicolumn{3}{|c|}{$\begin{array}{c}\text { Tabela } 6 \\
\text { Distribuição dos doentes segundo os fatores de } \\
\text { melhora da dor }\end{array}$} \\
\hline Fatores de melhora & $\mathbf{n}$ & $\%$ \\
\hline Repouso & 69 & 44,5 \\
\hline Não havia & 24 & 15,5 \\
\hline Postura & 23 & 14,8 \\
\hline Exercícios & 20 & 12,9 \\
\hline Massageamento & 13 & 8,4 \\
\hline Outras & 24 & 15,5 \\
\hline
\end{tabular}

Havia relato de piora da dor com os exercícios em $65,9 \%$ dos doentes, com condições estressantes em $61,9 \%$ e com determinadas posturas, em 16,8\% (Tabela 7).

\begin{tabular}{|c|c|c|}
\hline \multicolumn{3}{|c|}{$\begin{array}{c}\text { Tabela } 7 \\
\text { Distribuição dos doentes segundo fatores de piora da dor }\end{array}$} \\
\hline Fatores de piora & $\mathrm{n}$ & $\%$ \\
\hline Exercícios & 102 & 65,9 \\
\hline Estresse psíquico & 96 & 61,9 \\
\hline Não havia & 26 & 16,8 \\
\hline Postura & 26 & 16,8 \\
\hline Clima & 10 & 6,5 \\
\hline Outras & 16 & 10,3 \\
\hline
\end{tabular}

Todos os doentes foram submetidos a tratamento farmacológico que consistiu no uso de medicação neuroléptica (fenotiazinas, antidepressivos tricíclicos) e analgésicos antiinflamatórios não-hormonais (AAINHs) e a tratamento fisiátrico. Para 1/3 dos doentes foram prescritos medicamentos miorrelaxantes diazepínicos $(29,6 \%)$, baclofeno $(14,9 \%)$, analgésicos morfínicos $(14,9 \%)$, carbamazepina $(22,2 \%)$ e outros agentes $(13,7 \%)$. O resultado do tratamento foi considerado excelente quando ocorreu melhora de mais de $75 \%$ da dor original, bom quando a melhora variou de $51 \%$ a $75 \%$, regular quando de $25 \%$ a $50 \%$ e mau quando inferior a $25 \%$.

O tratamento farmacológico/fisiátrico proporcionou alívio substancial da dor em 25,2\% dos doentes, melhora considerável em $36,8 \%$, alguma melhora em $20,0 \%$ e piora ou melhora pouco expressiva em $18,1 \%$ dos doentes (Tabela 8).

\begin{tabular}{lcr}
\hline \multicolumn{2}{c}{$\begin{array}{c}\text { Tabela } 8 \\
\text { Resultado do tratamento farmacológico e fisiátrico } \\
\text { doentes }\end{array}$} \\
\hline Resultados & $\mathbf{n}$ & $\%$ \\
\hline Bom & 28 & 36,8 \\
Excelente & 39 & 25,2 \\
Regular & 31 & 20,0 \\
Mau & 57 & 18,0 \\
\hline Total & $\mathbf{1 5 5}$ & $\mathbf{1 0 0}$ \\
\hline
\end{tabular}

Três doentes foram submetidos à rizotomia percutânea por radiofreqüência para o tratamento da dor segmentar: o resultado foi bom em um doente, ocorreu piora expressiva em outro e outro ainda apresentou discreta melhora. A lesão do trato de Lissauer do corno posterior da medula espinal foi indicada para o tratamento de 21 doentes. Proporcionou resultado excelente em 12 doentes, resultado bom em dois, regular em três e mau em quatro. A estimulação elétrica epidural com eletródio implantável foi indicada em 42 doentes e proporcionou resultados excelentes em 13, resultados bons em 4, regulares em 3 e insatisfatórios em 22 . Foi realizada uma aplicação epidural de medicação morfínica, visando ao tratamento da dor em 6 doentes; o resultado foi considerado bom em 2 e insatisfatório em 4 (Tabela 9).

O período de acompanhamento variou entre 1 e 264 meses (mediana $=28$ meses).

\section{Discussão}

A lesão do sistema nervoso central (SNC) e periférico (SNP) é causa freqüente de dor. As afecções neuropáticas foram a causa de assistência em $24 \%$ dos 


\begin{tabular}{|c|c|c|c|c|c|c|c|c|c|}
\hline \multirow[b]{2}{*}{ Procedimentos } & \multicolumn{8}{|c|}{$\begin{array}{l}\text { Tabela } 9 \\
\text { sultados dos procedimentos cirúrgicos realizados }\end{array}$} & \multirow[b]{3}{*}{ Total } \\
\hline & \multicolumn{8}{|c|}{ Resultados } & \\
\hline & & xcelente & & Bom & & Regular & & Mau & \\
\hline Morfina peridural & & - & 2 & $(33,3 \%)$ & & - & 4 & $(66,6 \%)$ & 6 \\
\hline Estimulação elétrica epidural & 13 & $(31,0 \%)$ & 4 & $(9,5 \%)$ & 3 & $(7,1 \%)$ & 22 & $(52,3 \%)$ & 42 \\
\hline Lesão do trato de Lissauer e do CPME & 12 & $(57,1 \%)$ & 2 & $(9,5 \%)$ & 3 & $(14,3 \%)$ & 4 & $(19,0 \%)$ & 21 \\
\hline Rizotomia percutânea por radiofreqüência & & - & 1 & $(33,3 \%)$ & 1 & $(33,3 \%)$ & 1 & $(33,3 \%)$ & 3 \\
\hline
\end{tabular}

doentes atendidos na Unidade de Triagem do Centro de Dor do Hospital das Clínicas da Faculdade de Medicina da Universidade de São Paulo durante o ano de $1996^{87}$.

A dor é freqüentemente incapacitante em doentes com lesões raquimedulares. As afecções raquimedulares são bastante prevalentes no nosso meio. Em $15,5 \%$ dos doentes atendidos nessa unidade, a dor era resultante de lesões da medula espinal e, em 3,8\% dos casos, de síndrome dolorosa pós-laminectomia. A dor neuropática foi a segunda causa de procura pelo centro de dor, sendo as lesões raquimedulares a segunda razão de ocorrência de dor neuropática quando se excluíram as cefaléias; só foram superadas pelas neuropatias periféricas. Traumatismos, neoplasias, processos inflamatórios e desmielinizantes, acidentes operatórios, afecções vasculares, degenerativas ou congênitas são, entre outras, causas de dor mielopática ${ }^{7,11,17,52}$ e de dor por radiculopatia da cauda eqüina ${ }^{4,86}$. A freqüência de síndromes álgicas, nessas entidades, varia de $6,4 \%$ a $100 \%$ dos $\operatorname{casos}^{11,17}$, sendo incapacitantes em $27 \%$ a $40 \%$ delas $^{38}$; cerca de $10 \%$ desses doentes necessitam de tratamento neurocirúrgico para seu controle ${ }^{86}$. Em nossa estatística, a lesão por penetração de projétil de arma de fogo foi a causa mais comum de mielopatia e de lesões da cauda eqüina, seguindo-se os traumatismos fechados e as lesões iatrogênicas durante a execução de procedimentos cirúrgicos. Nos EUA, ocorrem, ao ano, 29 a 50 casos de traumatismos raquimedulares para cada 1 milhão de habitantes. Naquele país, em $50 \%$ dos casos, a causa é acidente de automóvel, em $15 \%$ a 20\%, acidentes domésticos e, em 10\% a 15\%, atos de violência. Admite-se que lá existam 72 casos de mielopatas traumáticos para cada 100 mil habitantes ${ }^{69}$. Essa condição predomina nos indivíduos do sexo masculino e nos jovens. A dor manifesta-se imediatamente após a lesão em $31 \%$ dos doentes, mantém-se dois anos após em $8 \%$ e apresenta modificação quanto à intensidade e características em $7 \%$ deles. A dor ocorre em $28 \%$ dos doentes com esclerose múltipla ${ }^{37}$. A esclerose múltipla é mais freqüente no sexo feminino; nos países do hemisfério norte, ocorrem 1 a 3 casos a cada 100 mil habitantes ao ano; o pico de ocorrência manifesta-se aos 30 anos de vida ${ }^{37}$. Os tumores podem ser causa de dor mielopática ou de dor radicular; a dor segmentar é mais comum em casos de lesões intramedulares ${ }^{43}$. A siringomielia costuma expressar-se em doentes com 28 a 41 anos de idade ${ }^{77}$, e ocorre dor em $50 \%$ desses doentes. A dor, às vezes, é o sintoma inaugural dessa condição $0^{77}$. Aproximadamente de 3\% a $5 \%$ dos doentes desenvolve dor neuropática após cordotomias $^{84}$.

Na presente casuística, $65,2 \%$ dos doentes era do sexo masculino. Esse dado está de acordo com o apresentado por Teixeira e cols. ${ }^{87}$, em 1999, que verificaram que na Unidade de Triagem do Centro de Dor do Hospital das Clínicas da Faculdade de Medicina da Universidade de São Paulo a dor por lesão raquimedular predominou nos indivíduos do sexo masculino (62,5\%); as mielopatias predominaram no sexo masculino e a síndrome dolorosa pós-laminectomia, no feminino. Essa distribuição quanto ao sexo, possivelmente, é relacionada à elevada prevalência de casos de lesões por traumatismos físicos acidentais ou agressões, responsáveis por $58,7 \%$ das lesões nos doentes avaliados na presente casuística. A mediana das idades dos doentes, na presente casuística, foi 47,1 anos, ou seja, a dor manifesta-se e incapacita indivíduos em plena fase produtiva.

As razões que justificam a ocorrência da dor em casos de lesões raquimedulares são motivos de muitas controvérsias. Não existem conceitos ou definições que possam ser aplicados a todas as síndromes dolorosas. Além disso, a intensidade da dor e a expressão do sofrimento diferem muito de um indivíduo para outro e nas diferentes espécies animais. Um grande número de fatores individuais, ambientais, incluindo os aspectos raciais, sociais, culturais, religiosos, filosóficos, experiências anteriores e o estado mental dos indivíduos pode exercer efeito exacerbador ou minimizador da expressão nociceptiva ${ }^{68}$. A dor nas lesões raquimedulares pode ser conseqüência de alterações anatomofuncionais das estruturas de suporte ou das vísceras, ou ter origem psicogênica, mas, geralmente, é neurogênica ${ }^{11,19,75}$. Portanto, pode ser decorrente de estímulos nociceptivos, sensibilização das unidades 
nociceptivas, geração de potenciais ectópicos nas estruturas nervosas periféricas ou outras desaferentadas e hipoatividade do sistema supressor da dor ${ }^{19}$. É provável que unidades noradrenérgicas e serotoninérgicas participem no mecanismo da ansiedade e depressão, fenômenos que, normalmente, estão associados à dor crônica ${ }^{5,19}$. Embora a experiência dolorosa implique na participação dos sistemas supraespinais, existe considerável integração da informação nociceptiva nos segmentos espinais ${ }^{71}$. À medida que se desloca rostralmente no neuroeixo, a redundância anatômica das vias sensitivas aumenta de modo significativo e a especificidade reduz-se.

A síndrome dolorosa por desaferentação é conhecida há várias décadas, especialmente depois da apresentação dos casos de síndrome talâmica por Dejerine e Roussy, em $1906^{19}$. Segundo Pagni ${ }^{52}$, em 1983, Riddoch apresentou o conceito de dor central, como: "Dor espontânea ou reação excessiva à estimulação objetiva, incluindo as disestesias e sensações desagradáveis, resultantes de lesões relacionadas com o SNC". Segundo Tasker e cols. ${ }^{70}$, a dor por desaferentação é a "resultante de lesões nervosas". Sweet ${ }^{69}$ define a dor por desaferentação como aquela que se "apresenta em doentes com lesões do SNP, medula espinal, tronco encefálico e encéfalo". Para a gênese da dor neurogênica, concorrem mecanismos periféricos e centrais ${ }^{19}$. O estresse, o medo, a ansiedade e a duração da dor interferem nos mecanismos de ativação do sistema modulador da analgesia ${ }^{19}$. A depressão e a ansiedade interagem na percepção da dor, via mecanismos inibitórios e facilitatórios ainda não elucidados adequadamente. As endorfinas e outros neuromoduladores da nocicepção são liberados quando a dor é muito intensa e há estresse.

Em condições normais, a integridade das vias nociceptivas no SNP e SNC e dos centros de processamento e supressão da dor é necessária para que o fenômeno nociceptivo manifeste-se normalmente ${ }^{19}$. A substância cinzenta do corno posterior da medula espinal (CPME) e o trato de Lissauer representam o primeiro centro de integração dos sistemas aferentes primários no neuroeixo ${ }^{76}$. A ação dos neurotransmissores excitatórios, liberados pelas terminações proximais dos aferentes primários, sofre influência de sistemas neuronais excitatórios e inibitórios segmentares e supra-segmentares no CPME. É provável que, na dependência da modulação da nocicepção segmentar, a informação nociceptiva seja ou não transferida pelos neurônios de segunda ordem para centros rostrais do neuroeixo. Em neuropatias radiculares, possíveis de ocorrer em casos de traumatismos raquimedulares, neoplasias e iatrogenias, instala-se hiperatividade neuronal na lâmina $\mathrm{V}$ do CPME que se torna progressivamente mais intensa no transcurso do tempo ${ }^{19,51}$ e é associada à expansão do campo receptivo nos neurônios aí sediados e nos núcleos dos tratos dos funículos posteriores ${ }^{19}$. A desinibição das unidades talâmicas nociceptivas, a sensibilização das sinapses centrais, a reorganização sináptica, a excitação celular resultante dos produtos de degradação neuronal durante o processo de degeneração e a proliferação das células gliais no $\mathrm{CPME}^{30}$ contribuem para a gênese da dor nessa eventualidade. Mecanismos similares justificam a dor segmentar em doentes com mielopatias. Essa é atribuída à interrupção do trato de Lissauer. A dor nos segmentos desaferentados distais é atribuída à hiperatividade neuronal no $\mathrm{CPME}^{31,32}$, à modificação do padrão da aferência dos estímulos sensitivos ao tálamo ${ }^{7} \mathrm{e}$ à hiperatividade dos núcleos sensitivos do tronco encefálico e do córtex cerebral; ocorrem modificações anatômicas, eletrofisiológicas e neuroquímicas significativas nas unidades que compõem o CPME $^{31,32,51,70}$, caracterizadas por expansão dos campos receptivos e hiperatividade neuronal ${ }^{30} \mathrm{e}$ maior afluência de aferências inibitórias contralaterais, influenciadas por vias descendentes. O comprometimento dos tratos rostrocaudais supressores que ativam neurônios inibitórios segmentares contribui para a expansão do campo receptivo nas unidades neuronais da lâmina $\mathrm{V}$ do CPME e para a redução da proporção dos neurônios excitados diante da estimulação não-nociceptiva ${ }^{22}$. As anormalidades eletroencefalográficas e o aumento da atividade espontânea no núcleo grácil ${ }^{17}$ são também identificadas. A lesão desse núcleo causa aumento do campo receptivo das unidades neuronais desaferentadas no complexo ventrobasal do tálamo ${ }^{28,29}$. As zonas-gatilho observadas em doentes mielopatas são associadas ao aumento da atividade excitatória prolongada de neurônios que recebem aferência de áreas não-desaferentadas; significa que existem reorganização e aumento da área de projeção dos aferentes nociceptivos nessas unidades neuronais ${ }^{19}$. A estimulação dos tratos discriminativos na medula espinal reduz a hiperatividade neuronal espontânea nos núcleos mediai $^{39}$ e no complexo ventrobasal ${ }^{74}$ do tálamo. $\mathrm{Ob}$ servou-se haver redução da concentração de catecolaminas e substância P no CPME em casos de secção de medula espinal em animais de experimentação ${ }^{8}$.

Em nossa casuística, ocorreu maior freqüência de casos com lesões no segmento torácico da medula espinal, seguindo-se os do segmento lombar e cervical. Tasker e Dostrovsky ${ }^{70}$ observaram que a dor é mais freqüente quando a lesão ocorre nos segmentos mais caudais da medula espinal; manifestou-se dor em $15 \%$ dos casos de lesões cervicais, em $24 \%$ de casos das lesões torácicas e em $51 \%$ de casos das lesões torácicas caudais e lombares. O período de duração da síndrome álgica variou de 1 a 564 
meses e teve mediana de 61,7 meses, o que traduz o prolongado sofrimento dos nossos doentes. Na casuística de Tasker e Dostrovsky ${ }^{70}$, a dor manifestou-se imediatamente após a lesão em $31 \%$ dos doentes e, ao longo dos dois primeiros anos, em $8 \%$.

Em nossa casuística, em 75,4\% dos casos, a dor acometeu, pelo menos, os membros inferiores. A maior prevalência de dor nos membros inferiores é devida ao fato de haver maior freqüência de doentes com lesões da medula espinal torácica ou da cauda eqüina ou da medula lombar. Ocorreu, também, predomínio da sensação de dor fantasma nos segmentos desaferentados em $88,3 \%$ dos doentes, seguindo-se a ocorrência de síndrome dolorosa miofascial, observada em $25 \%$ dos casos e da dor segmentar em $15,4 \%$. A dor por desaferentação associada à hiperpatia e alodinia pode apresentar-se no limite entre a zona de hipoestesia e de sensibilidade normal, mimetizando dor radicular (dor na faixa de transição), ou como dor nas áreas distais à lesão (dor fantasma $)^{70}$. A dor na faixa de transição é, geralmente, simétrica e bilateral ${ }^{35}$. A estimulação mecânica das regiões proximais à desaferentada, possivelmente, ativa estruturas corticais e subcorticais supressoras ${ }^{35}$. O fenômeno fantasma, caracterizado por sensação de postura e movimentos anormais dos segmentos desaferentados, é freqüente e, muitas vezes, surge entre os $6^{\circ}$ e o $12^{\circ}$ - meses após a lesão medular ${ }^{70}$. A dor fantasma localiza-se freqüentemente na região sacral e na porção distal dos membros inferiores, mas pode apresentar-se em qualquer região desaferen$\operatorname{tada}^{15,16}$. Cerca de $5 \%$ dos doentes apresentam dor visceral, caracterizada pela sensação de flatulência, ardor, ou cólica referida no abdômen ou na pelve.

Ocorreu predomínio da sensação de queimor ou queimor e choque. A dor neuropática é espontânea e pode ser desencadeada por estímulos inócuos. É descrita como queimor, pontada, adormecimento, formigamento ou sensações vagas estranhas. Geralmente é de início tardio e referida em áreas nas quais se observam alterações da função neurológica ${ }^{35}$. Queimor e formigamento constantes ocorrem em aproximadamente 50\% dos doentes $7,16,52,75$; aperto, torção ou câimbra em $25 \%$ e pontada em $20 \%{ }^{70}$. A sensação de choque é mais comum nas lesões parciais dos segmentos caudais da medula espinal ${ }^{57}$. Pode manifestar-se continuadamente ou em crises durando poucos minutos ${ }^{15,16,70}$.

Nesses doentes, havia relato de melhora da dor com o repouso em $44,5 \%$, com adaptação da postura em $14,8 \%$ e com exercícios em $12,9 \%$. Em $8,4 \%$ das vezes, o massageamento das áreas justapostas àquelas em que a dor era referida resultava em alívio da dor. Havia relato de piora da dor com a atividade física em $65,9 \%$ dos nossos casos, seguindo-se os estresses psíquicos, as modificações da postura e as alterações climáticas. A dor pode ser desencadeada por automatismos medulares, modificações da pressão atmosférica, umidade ambiental e emoções. Além do agravamento da dor, náuseas, hiperemia cutânea, cefaléia, transpiração profusa, piloereção e taquicardia podem ser desencadeados pela distensão vesical e retal ${ }^{13}$. Esse quadro é atribuído à transferência da informação nociceptiva pelas estruturas do sistema nervoso neurovegetativo ${ }^{6}$. Em casos de lesão da cauda eqüina, síndromes dolorosas rebeldes são comuns ${ }^{7,10,15,16,52,54,70,75}$. A dor pseudotabética pode ser observada em casos de síndrome dolorosa pós-laminectomia para o tratamento de hérnia discal lombar.

O tratamento da síndrome dolorosa envolve interações psicossociais e biológicas. Se consideramos a complexidade das vias e mecanismos de modulação nociceptiva e os fenômenos comportamentais complexos associados às síndromes dolorosas crônicas, entende-se a dificuldade de controlar a dor por desaferentação. Sistemas neuronais supra-espinais permitem ao organismo utilizar a experiência passada para controlar a sensibilidade nos vários setores do neuroeixo e intervir de modo variável e determinado. Medicamentos, medicina física, terapia ocupacional, psicoterapia e condicionamento físico devem ser aplicados nos doentes com dor crônica, com a finalidade de melhorar o rendimento funcional e o desempenho psíquico e social dos doentes ${ }^{2,68}$. A eliminação dos fatores que originam a dor, o controle da ansiedade, o esclarecimento das situações, a reabilitação precoce e o retorno às atividades cotidianas são fundamentais ${ }^{2}$. O tratamento da dor resultante de lesão raquimedular é baseado na erradicação do agente causal e dos fatores agravantes. Entretanto, freqüentemente, a erradicação dos agentes etiológicos não elimina a dor neuropática. Procedimentos ortopédicos e fisiátricos são úteis no tratamento da dor resultante de lesões osteoarticulares e ligamentares ${ }^{71}$. O bloqueio anestésico dos pontos-gatilho miofasciais é eficaz no tratamento da síndrome dolorosa miofascial $^{11,16,54}$. Na fase aguda dos doentes com traumatismos raquimedulares, os analgésicos antiinflamatórios e os agentes morfínicos podem ser utilizados, uma vez que os fenômenos inflamatórios contribuem expressivamente para a ocorrência da dor. Na presente casuística, o tratamento farmacológico e fisiátrico beneficiou sensivelmente $62 \%$ dos doentes. Os analgésicos, morfínicos ou não, com raras exceções, são ineficazes no tratamento da dor por desaferentação ${ }^{2}$. Anticonvulsivantes, antineurálgicos, miorrelaxantes, antidepressivos e neurolépticos são habitualmente receitados para o tratamento das síndromes dolorosas neuropáticas. Todos os doentes haviam sido medicados com analgésicos antiinflamatórios, fenotiazinas e antidepressivos tricíclicos. Foi demonstrado que antidepressivos 
tricíclicos e fenotiazinas são úteis no tratamento da dor mielopática neuropática constante ${ }^{57}$. As fenotiazinas apresentam ação ansiolítica, analgésica e sedativa ${ }^{18,53}$. Os resultados são melhores quando associados com antidepressivos $^{40}$. O efeito analgésico dos antidepressivos é atribuído ao bloqueio da recaptação de serotonina e noradrenalina nas vias supressoras da dor que, do tronco encefálico, projetam-se nas unidades neuronais do $\mathrm{CPME}^{6,14,67,79} \mathrm{e}$ a mecanismos independentes da ação sobre esses neurotransmissores, incluindo o efeito sedativo, ansiolítico, miorrelaxante, antiinflamatório, bloqueador dos canais do cálcio e de prostaglandinas, e de receptores de histamina e encefalinas ${ }^{9,40,71}$. Os anticonvulsivantes antineurálgicos são úteis para o tratamento da dor paroxística ${ }^{3,14,21}$. Essa foi a razão da prescrição de carbamazepina, isolada ou associadamente a outros anticonvulsivantes, em $2,2 \%$ dos doentes do presente caso que apresentavam significativo componente de dor paroxística.

Em seis doentes da presente casuística, foi instilada solução de morfina com anestésicos locais no compartimento epidural raquidiano. O bloqueio anestésico peridural usualmente não beneficia doentes com dor mielopática, mas é útil para o tratamento da dor por irritação de raízes nervosas ${ }^{11}$. O bloqueio anestésico da cadeia neurovegetativa simpática e/ou simpatectomia também é ineficaz ${ }^{11}$.

Muitas intervenções cirúrgicas foram propostas para o tratamento da dor mielopática e da dor por lesão da cauda eqüina. A rizotomia percutânea por radiofreqüência foi utilizada para o tratamento da dor segmentar em três doentes da presente casuística; apenas um doente apresentou benefício com a intervenção. A neurólise química e a rizotomia também não beneficiam doentes com dor mielopática e podem agravar a desaferentação ${ }^{10,11,15}$. A cordotomia anterolateral e a mielotomia, propostas com o objetivo de reduzir a transferência rostracaudal das informações nociceptivas segmentares ${ }^{2,10,80}$, proporcionam resultados controversos. A cordotomia melhora a dor aguda em pontada e em câimbra e a dor provocada por estímulos aplicados na zona-gatilho, mas não o queimor constante referido nos membros inferiores e na região perineal; pode melhorar a dor radicular causada por lesões na cauda eqüina, mas não o resultante da lesão da medula torácica ${ }^{80}$. Por outro lado, a cordotomia indicada para outras condições é causa de dor em cerca de $20 \%$ dos doentes ${ }^{80}$. A cordotomia melhorou a dor intermitente e lancinante de $70 \%$ dos doentes e a dor constante em $47 \%$ dos doentes com lesão mielorradicular, na casuística de Tasker e Dostrovsky ${ }^{70}$. Ocorreu melhora da dor em $70 \%$ dos doentes com lesão de cone medular e da cauda eqüina, e em apenas $40 \%$ com lesão nas porções rostrais da medula espinal.
Resultados semelhantes foram relatados por outros autores ${ }^{15}$. A mielotomia transversa, realizada dois a três segmentos rostrais aos da lesão, pode aliviar, pelo menos temporariamente, a dor radicular ou do território de transição ${ }^{61}$. A talamotomia e a mesencefalotomia foram indicadas para o tratamento da dor neuropática ${ }^{35}$; entretanto, os resultados foram freqüentemente insatisfatórios a longo prazo $^{80}$. A ablação da área sensitiva principal proporciona resultados insatisfatórios ${ }^{80} \mathrm{e}$ a lobotomia frontal ${ }^{80}$, bons resultados, mas com o risco elevado de gerar seqüelas mentais graves ${ }^{15,52}$. A cingulotomia proporciona melhores resultados porque não altera as funções mentais, porém o número de publicações a respeito é insignificante ${ }^{80}$.

A eliminação da hiperatividade neuronal segmentar, no $\mathrm{CPME}^{76}$, pela técnica da lesão do CPME e do trato de Lissauer ${ }^{23,26,48,55,66,81}$, é eficaz no tratamento da dor por desaferentação, incluindo a dor mielopática e a causada por lesão de raízes nervosas ${ }^{45,59,72}$. Na presente casuística, esse procedimento proporcionou melhora significante ou alívio da dor em $2 / 3$ dos doentes. Aceitase que essa intervenção proporcione resultados iniciais satisfatórios em $85 \%$ a $100 \%$ dos casos e beneficia, a longo prazo, $46 \%$ a $80 \%$ dos doentes ${ }^{20,58,59}$. Powers e cols. ${ }^{23}$, entretanto, não observaram melhora da dor segmentar em dois e da dor fantasma em cinco dos nove doentes tratados por essa cirurgia. Na casuística de Friedman e Bullit ${ }^{20}$, esse procedimento proporcionou alívio significativo da dor segmentar em $80 \%$ dos doentes com dor unilateral, em $90 \%$ dos casos de dor lombar e sacral $^{60}$. Thomas e Jones ${ }^{72}$ não verificaram melhora em doentes com dor mielopática e observaram piora do déficit neurológico em $50 \%$ dos doentes durante o período pós-operatório. Segundo Nashold ${ }^{56}$, esse procedimento alivia especialmente a dor gerada pela estimulação das zonas-gatilho. Os resultados em casos de síndrome dolorosa pós-laminectomia são precários ${ }^{56,60}$ pois, além de não beneficiar os doentes, inúmeras complicações neurológicas foram descritas depois da execução desse procedimento nesses doentes, incluindo-se, dentre elas, a hipoestesia na região genital e nos membros inferiores, os déficits motores, a incontinência esfincteriana e a impotência sexual ${ }^{45,60}$.

A estimulação elétrica da medula espinal (EME) e do encéfalo é, ainda, amplamente empregada para o tratamento da dor ${ }^{22,36,56,61,62,64,78}$, pois proporciona ativação antidrômica dos funículos posteriores ${ }^{12,62,63}$ ou das vias de condução inespecíficas da medula espinal ${ }^{62}$, ativa os circuitos inibitórios corticotalâmicos ${ }^{41} \mathrm{e}$ as unidades neuronais do sistema supressor do tronco encefálico que originam tratos rostrocaudais que inibem a atividade das unidades nociceptivas segmentares na medula espina ${ }^{33}$. A participação das vias encefalinérgicas e endorfinérgicas no mecanismo da analgesia induzida pela estimulação da 
medula espinal é discutível ${ }^{73}$. Parece ser importante o papel representado pelas vias que utilizam noradrenalina, dopamina, somatostatina, colecistoquinina, peptídio vasoativo intestinal, neurotensina e substância P no mecanismo de ação analgésica da estimulação elétrica da medula espinal ${ }^{73}$. A estimulação dos núcleos talâmicos específicos $^{34}$, cápsula interna ${ }^{1}$, substância cinzenta periventricular, substância cinzenta periaquedutal mesencefálica, núcleo caudado, septo e região parabraquial pontomesencefálica ${ }^{28}$ parecem ser também úteis no tratamento da dor neuropática; a estimulação da substância periaquedutal mesencefálica, que induz a liberação de neurotransmissores morfínicos no líquido cefalorraquidiano, é útil no tratamento da dor por nocicepção ${ }^{25}$. Em 40,5\% dos casos da presente casuística, a EME proporcionou melhora satisfatória. A EME proporciona melhora em $50 \%$ dos casos durante o primeiro ano. Após um ano de seguimento, apenas $20 \%$ dos doentes mantêm a dor controlada ${ }^{16,57}$. Em casos de lesão da cauda eqüina e do cone medular, a EME proporciona melhora prolongada significativa em poucos $\operatorname{casos}^{29,57}$. A EME beneficia significativamente e imediatamente 50\% dos doentes com lesão da cauda eqüina e, a lesão do CPME, proporção similar ${ }^{27,60}$. A EME não alivia a dor mielopática nas regiões totalmente desaferentadas ${ }^{46,57}$. Nesses casos, o insucesso da EME deve estar relacionado com a acentuada degeneração das vias sensitivas que, da medula espinal projetam-se no encéfalo, limitando, desse modo, a ativação das unidades supressoras segmentares e supra-segmentares ${ }^{57}$.

A EME, portanto, está indicada no tratamento da dor mielopática em doentes em que a lesão das vias sensitivas é parcial ${ }^{36}$ e a lesão do CPME, em casos de lesões completas. O número de complicações, contudo, é maior após a lesão do CPME em doentes com preservação da função neurológica, principalmente quando realizada na medula torácica e lombossacral. Essas complicações referem-se, principalmente, ao comprometimento dos funículos posteriores e do trato espinotalâmico ${ }^{49,56,60,75}$. Por esse motivo, dita técnica deve ser reservada para doentes com grave comprometimento da função neurológica, tal como ocorre em paraplégicos com mielopatia ou com secção das raízes da cauda eqüina. O caráter pouco agressivo da estimulação do SNC faz com que essa técnica seja adequada para o tratamento de doentes com conservação da função neurológica.

Foram descritos casos isolados de melhora dos doentes com dor mielopática tratados por estimulação dos núcleos talâmicos sensitivos ${ }^{74}$, lemnisco medial e da substância periaquedutal mesencefálica ${ }^{24}$. A estimulação do tálamo, da cápsula interna e do lemnisco medial beneficia cerca de $1 / 3$ dos doentes ${ }^{62,65,82}$.

Conclui-se que a dor em doentes com lesões raquimedulares é freqüentemente rebelde ao tratamento, e que a seqüência de prescrição de analgésicos e psicotrópicos, medicina física e, quando necessárias, medidas cirúrgicas, incluindo a estimulação elétrica epidural e a lesão do trato de Lissauer e do corno posterior da medula espinal, proporciona melhora satisfatória em significante número de casos .

\section{Referências}

1. ADAMS JKE, HOSOBUCHI Y, FIELDS H: Estimulation of internal capsule for relief of chronic pain. J Neurosurg 41:740-4, 1974

2. ADDISON RG: Chronic pain syndrome. Am J Med 10:54-8,1984.

3. ALBERT MZ: Treatment of pain in multiple sclerosis. Preliminary report. N Engl J Med 28:1395, 1969.

4. AJURIAGUERRA DJ: La douleur dans les affections du système nerveux central. Paris, Doin, 1937.

5. ARNER S, MEYERSON BA: Lack of analgesic effect of opioids in neurophatic and idiophatic form of pain. Pain 33:11-23, 1988.

6. ASBERG M, THOREN P, TRASKMAN L, BERTILSSON L, RINGBERGER V: Serotonin depression a biomechanical sugroup within the affective disorders? Science 191:478-80, 1976.

7. BEDBROOK GM: Injuries of the thoracolumbar spine with neurological symptoms. In: Vinken PJ, Bruyn GW (eds): Handbook of Clinical Neurology. Amsterdam, North-Holland Publishing Company, 1976, vol 25, pp 437-466.

8. BERIC A, DIMITRIJEVIC MR, LINDBLOM O: Central dysesthesia syndrome in spinal cord injury patients. Pain 34:109-16, 1988.

9. BLOEDEL JR: The substrate for integration in the central pain pathways. Clin Neurosurg 16:194-228, 1976.

10. BOTNEY M, FIELDS HZ: Amitriptylline potentiates morphine analgesia in a direct action on the central nervous system. Ann Neurol 13:160-4, 1983.

11. BOTTERELL EH, CALLAGHAN GC, JOUSSE AT: Pain in paraplegia: clinical management and surgical treatment. Proc R Soc Med 47:281-8, 1954.

12. BURCHIEL KJ, RUSSEL LC: Spontaneous activity of ventral root axons following peripheral nerve injury. J Neurosurg 62:408-13, 1985.

13. BURKE DC, WOODWARD JM: Pain and phantom sensation in spinal paraplegia. In: Vinken PJ, Bruyn GW (eds): Handbook of Clinical Neurology. Amsterdam, North-Holland Publishing Company, 1976, vol 26, pp 489-499.

14. CIONI B, MEGLIO M: Epidural recording of electrical events produced in the spinal cord by segmental ascending and descending volleys. Appl Neurophysiol 49:315-26, 1986.

15. COX GB, CHAPMAN CR: Multivariate analysis of pain data. In: Bonica JJ, Albe Fessard DG (eds): Advances in Pain Research and Therapy. New York, Raven Press, 1976, vol 1, pp 369-373.

16. DALESSIO DJ: Chronic pain syndromes and disordered cortical anhibition. Effects of tricyclic compounds. Dis Nerv Syst 28:325-8, 1967.

17. DAVIS L, MARTIN J: Studies upon spinal cord injuries. Nature and treatment of pain. J Neurosurg 4:483-91, 1947. 
18. DAVIS R, LENTINI R: Transcutaneous nerve stimulation for treatment of pain in patients with spinal cord injury. Surg Neurol 4:100-1, 1975.

19. DEJERINE J, ROUSSY G: Le sindrome thalamique. Rev Neurol (Paris) 14:521-32, 1906.

20. DEVOR M: The pathofhisiology of the damaged peripheral nerves. In: Wall PD, Melzack R (eds): Textbook of Pain. Edimburgh, Churchil Livingstone, 1989, pp 63-81.

21. DONOVAN WH, DIMITRIJEVIC MR, DAHM L, DIMITRIJEVIC M: Neurophysiological approaches to chronic pain following spinal cord injury. Paraplegia 20:135-46, 1982.

22. DOSTROVSKY JO, MILLAR D: Receptive fields of gracile neurons after transduction of he dorsal columns. Exp Neurol 56:610-21, 1977.

23. DUBULSSON D: Nerve root damage and arachnoiditis. In: Wall PD, Melzack R (eds): Textbook of Pain. Edimburgh, Churchil Livingstone, 1989, pp 544-65.

24. DUNDEE JW, LOVE WJ, MOORE J: Alteration in response to somatic pain associates with anaesthesia. Further studies with phenothiazine derivates and similar drugs. Br J Anaesthesiol 35:597-609, 1963.

25. FIELDS HL: Neurophysiology of pain and pain modulation. AM J Med 10:54-68, 1984.

26. FIELDS HL, BASBAUM AL: Endogenous pain control mechanism. In: Wall PD, Melzack R (eds): Textbook of Pain. Edimburgh, Churchil Livingstone, 1989, pp 206-17.

27. FRIEDMAN AH, BULLIT E: Dorsal root entry zone lesions in the treatment of pain following brachial plexus avulsion, spinal cord injury and herpes zoster. Appl Neurophysiol 51:164-69, 1988.

28. FRIEDMAN AH, NASHOLD BS Jr: DREZ lesions for relief of pain related to spinal cord injury. $J$ Neurosurg 65:465-9, 1986.

29. GIBSON JC, WHITE LE Jr: Denervation hyperphatia. A convulsive syndrome of the spinal cord responsive to carbamazepina therapy. J Neurosurg 35:287-90, 1971.

30. GILDERBERG PL, MORTHY KSK: Modification of thalamic evoked activity by dorsal column stimulation. Acta Neurochir (Suppl) (Wien) 24:159-61, 1977.

31. HITCHCOCK E: Stereotatic trigeminal tractotomy. Ann Clin Res 2:131-5, 1970.

32. HOSOBUCHI Y: The current status analgesic brain stimulation. Acta Neurochir (Wien) (Suppl) 30:21927, 1980

33. HOSOBUCHI Y, ROSSIER J, BLOOM FE, GUILLERMIN $R$ : Stimulation of human periaqueductal gray of pain for pain relief increases imunoreactive beta-endorfin in ventriculary fluid. Science 203:279-81, 1979

34. HYNDMAN OR: Lissauer's tract section. A contribution to chordotomy for the relief of pain (preliminary report). J Int Coll Surg 5:394-400, 1942.

35. ISHIJIMA B, SHOIMOJI K, SHIMIZU H, TALAHASHI $\mathrm{H}$, SUZUKI I: Lesions of spinal and trigeminal dorsal root entry zone for deafferentation pain. Experience of 35 cases. Appl Neurophysiol 51:175-87, 1988.

36. KATAYAMA Y, TSUBOKAWA T, HIRAYAMA T, YAMAMOTO T: Pain relief following stimulation of the pontomesencephalic parabrachial region in humans: brain sites for nonopiate-medicated pain control. Appl Neurophysiol 48:195-200, 1985.

37. KURTZKE JF: Epidemiology of multiple sclerosis. In: Vinken PJ, Bryn GW, Klawans HL (eds): Handbook of Clinical Neurology, Amsterdan, Elsevier, 1985, vol 47, pp 259-287.
38. LEVY WJ, GALLO C, WATTS C: Comparison of laser and radiofrecuency dorsal root entry zone lesions in cats. Neurosurgery 16:327-30, 1985.

39. LIGTH AR: Normal anatomy and physiology of the spinal cord dorsal horn. Appl Neurophysiol 51:78-88, 1988.

40. LOESER JD, WARD AA: Some effects of deafferentation on neurons of the cat spinal cord. Arch Neurol 17:629636, 1967.

41. LOESER JD, WARD AA, WHITE IE: Chronic deafferentation of human spinal cord neuron. J Neurosurg 29:48-50, 1968.

42. LONG DM, ERICKSON D, CAMPBELL J, NORTH R: Electrical stimulation of the spinal cord and peripheral nerves for pain control. A ten years experience. Appl Neurophysiol 44:207-17, 1981.

43. LAMID S, CHIA JK, KOHLI A: Chronic pain in spinal cord injury: comparison between inpatients and outpatients. Arch Phys Med Rehabil 66:777-8, 1985.

44. MANSURY L, SINDOU M: Physiology of pain at the spinal cord level: neurosurgical aspects. In: Carrea R (ed): Neurological Surgery. International Congress Series, Amsterdam, Excerpta Médica, 1978, vol 433, pp 257-63, 1978.

45. Mc CORMICK PC, STEIN BM: Spinal cord tumors in adults. In: Youmans JR (ed): Neurological Surgery, ed 4, Philadelphia, WB Saunders Co, 1996, pp 3102-22.

46. MEHLER WR, FEFERMAN ME, NAUTA WJH: Ascending axon degeneration following anterolateral cordotomy. An experimental study in monkey. Brain 83:718-51, 1960.

47. MELZACK R, LOESER JD: Phantom body pain in paraplegics: evidence for a central "pattern generating mechanism" for pain. Pain 4:195-210, 1978.

48. MELZACK R, WALL PD: Pain mechanism; a new theory. Science 150:971-9, 1965.

49. MEYERSON BA: Biochemistry of pain relief with intracerebral stimulation. Acta Neurochir (Wien) (Suppl) 30:229-37, 1980.

50. MEYERSON DA, BRODIE E, BINDEROLLI B: Possible neurohumoral mechanism in CNS stimulation for pain supression. Appl Neurophisiol 48:175-80, 1985.

51. MODESTI LM, WASZAK M: Firing patterns of cells in the human thalamus during dorsal column stimulation. Appl Neurophysiol 38:251-80, 1975

52. MONKS R, MERSKEY H: Psycotrophic drugs. In: Wall PD, Melzack R (eds): Textbook of Pain. Edimburgh, Churchil Livingstone, 1989, pp 702-21.

53. MORHLAND S, GEBHART G: Effects of local electrical stimulation and morphine microinjection in the periaqueductal gray of the rat mesencephalon on neuronal activity in the reticular formation. Brain Res 201:23-37, 1980.

54. MUNDINGER F, SALOMÃO JF: Deep brain stimulation in mesencephalic lemniscus medialis for chronic pain. Acta Neurochir (Wien) (Suppl) 30:245-58, 1980.

55. NASHOLD BS Jr: Deafferentation pain in man and animals as it relates to the DREZ operation. Can $\mathrm{J}$ Neurol Sci 15:5-9, 1988.

56. NASHOLD BS Jr: Introducing to Second International Symposium on Dorsal Root Entry Zone (DREZ) Lesions. Appl Neurophysiol 51:76-7, 1988.

57. NASHOLD BS Jr, BULLIT E: Dorsal root entry zone lesions to control central pain in paraplegics. J Neurosurg 55:414-9, 1991

58. NASHOLD BS Jr, FRIEDMAN H: Dorsal column stimulation for control of pain. Preliminary report on 30 patients. J Neurosurg 36:590-7, 1972. 
59. NASHOLD BS Jr, OSTDAHL RH: Dorsal root entry zone lesions for pain relief. J Neurosurg 51:59-69, 1979.

60. NASHOLD BS Jr, URBAN B, ZORUB DS: Phantom pain relief by local destruction of the substantia gelatinosa of Rolando. In: Bonica JJ, Albe Fessard DG (eds): Advances in pain and therapy. New York, Raven Press, vol 1, pp 959-63, 1976.

61. NASHOLD BS Jr, OSTDAHL RH: Pain relief after dorsal root entry zonelesions. Acta Neurochir (Wien) (Suppl) 30:383-9, 1980.

62. NASHOLD JD Jr, WILSON WP, SLAUGHTER DG: Sensations evoked by simulation in the midbrain of man. J Neurosurg 30:14-24, 1969.

63. OLVELMENT LEVITT J: Abnormal physiology of the dorsal horn as related to the deafferentation syndrome. Appl Neurophysiology 51:104-16, 1988.

64. PAGNI CA: Central pain and painful anesthesia. Prog Neurol Surg 8:132-257, 1976.

65. PATTS HV, PLEUVRY BJ: Interactions of morphine and methotrimeprazine in mouse and man with respect to analgesia, respiration and sedation. $\mathrm{Br}$ Anaesth J Anaest 55:437-41, 1983.

66. PETTIT, DUTAILLIS D, MESSIMY R, BORGES L: La psychochirurgie des algies irreductibles. Étude basée sur 57 cas. Sem Hop Paris 29:3893-903, 1953.

67. PINEDA: Complications of dorsal column stimulation. J Neurosurg 48:64-8, 1978.

68. POLLOCK LJ, BROWN M, BOSCHES B, FINKELMAN I, CHOA H, ARIEFF AJ, FINKLE FR: Pain below the level of injury of the spinal cord. Arch Neurol Psychiatry 65:319-22, 1951.

69. POOL JL: Posterior cordotomy for relief of phantom limb pain. Ann Surg 386-91, 1946.

70. POWERS SK, BARABARO NM, LEVY RM: Pain control with laser-produced dorsal root entry zone lesions. Appl Neurophysiol 51:243-54, 1988.

71. RICHARDSON RR, MEYER PR, CERULLO LJ: Neurostimulation in the modulation of intractable paraplegia in traumatic neuroma pain. Pain 25:7584, 1980.

72. RICHTER HP, SELTZ K: Dorsal root entry zone lesions for the control of deafferentation pain: experiences in ten patients. Neurosurgery 15:956-9, 1984

73. SAMMI M, MORINGLANE Jr: Thermocoagulation of the dorsal root entry zone for the treatment for intractable pain. Neurosurgery 15:953-6, 1984.

74. SARIS SC, VIEIRA JFS, NASHOLD BS Jr: Dorsal root entry zone coagulation for intractable sciatica. Appl Neurophysiol 51:206-11, 1988

75. SELIGMAN LG: Electrical stimulation of the spinal cord; a critical review. Pace 10:176-9, 1987.

76. SHEALY CM, MORTIMER Jr, RESWICK JB: Electrical inhibition of pain by stimulation of the dorsal columns. Preliminary clinical report. Anest Analg 46:489-91, 1967.

77. SICHEZ JP, CAPELLE L, DUFFAU H: Syringomyélie. In: Encycl Méd Chir, Neurologie, Paris, Elsevier, 1997, 17-077-A-10, pp 26.

78. SIEGFRED J: Introduction historique. Neurochirurgie (Sttuttg) (Suppl):5-10, 1976.

79. SIEGFRED J: Sensory thalamic neurostimulation for chronis pain. Pace 10:209-12, 1987.

80. SINDOU M: Étude la jonction radiculo medullairepostériere. La radiculotomie postériere selective dans la chirurgie de la douleur. Tese. Travail de l'Hospital Neurologique et de'l Unité de Recherchers de Physiopathologie de Sistéme Nerveux, 1972.
81. STERNBACH RA, JANOWSKY DS, HUEY IY, SEGAL DA: Effects of altering brain serotonin activity on human chronic pain. In: Bonica JJ, Albe Fessard D (eds): Advances in pain research and therapy. New York, Raven Press, 1976, vol 1, pp 601-6.

82. SWANSON DW, SWENSON WM, MARULA T, MCPHEE MC: Program for managing chronic pain. I. Program description and characteristic of patients. Mayo Clinic Proc 51:401-8, 1976.

83. SWEET WH: Deafferentation pain in man. Appl Neurophysol 51:117-27, 1988.

84. TASKER RR, DOSTROVSKY FO: Deafferentation and central pain. In: Wall PD, Melzack R (eds): Textbook of Pain. Edimburg, Churchil Livingstone, 1989, pp 154-80.

85. TASKER RR, ORGAN LW, HAWRYLYSHYN P: Deafferentation and causalgia. In: Bonica JJ (ed): Pain. New York, Raven Press, 1989, pp 305-29.

86. TEIXEIRA MJ: A lesão do trato de Lissauer e da substância cinzenta do corno posterior da medula espinal e a estimulação elétrica do sistema nervoso central para o tratamento da dor por desaferentação. Tese (Doutorado). Faculdade de Medicina da Universidade de São Paulo, 1990, pp 201.

87. TEIXEIRA MJ, MARCON RM, ROCHA RO, FIGUEIRÓ JB: Epidemiologia clínica da dor. Rev Med 78:3654, 1999.

88. TONELLI L, SETTI T, FALASCA AS, MARTIGNONI E, TROCIAE, CALCATERRA FM, MERLI GA, FACHINETTI $F$ : Investigation on cerebrospinal fluid opiods and neurotransmitters relates to spinal cord stimulation. Appl Neurophysiol 51:324-32, 1989.

89. TSUBUKAWA T, KATAYAMA Y, YAMAMOTO, HIRAYAMA T: Deaferentation pain and stimulation of the thalamic sensory relay nucleus: clinical and experimental study. Appl Neurophysiol 48:166-71, 1985.

90. WAISBROD H, HANSEN D, GERBERSHAGEN HV: Chronic pain in paraplegics. Neurosurgery 20:15, 1984.

91. WALL PD: The dorsal horn. In: Wall PD, Melzack R (eds): Textbook of Pain. Edimburgh, Churchill Livingstone, 1989, pp 102-11.

92. WALL PD, EGGER MD: Formation of new connections in adult brain after partial deafferentation. Nature 232:542-45, 1971

93. WALL PD, SWEET WH: Temporary abolition of pain in man. Science 155:108-9, 1967.

94. WATSON CPN, EVANS RJ, REED K, MERSKEY H, GOLDSMITH Z, WARSH J: Amitriptylline versus placebo in postherphetic neuralgia. Neurology (NY) 32:671-3, 1982.

95. WHITE JC, SWEET WH: Pain and Neurosurgeon. Springfield, Charles C Thomas, 1969.

96. YASKSH TL, FREDERICK WL: Kerr: Comments of his life with notes on his contributions of understanding of facial neuralgia. Appl Neurophysiol 51:65-75, 1985.

97. YOUNG RF, KROENING R, FULTON W, FELDMAN RA, CHAMBI I: Electrical stimulation of the brain in treatment of chronic pain. Experience over five years. J Neurosurg 62:389-96, 1985.

Original recebido em abril de 1999

Aceito para publicação em junho de 1999

\section{Endereço para correspondência:}

Manoel Jacobsen Teixeira

Rua General Mena Barreto, 756

CEP 01433-010 - São Paulo, SP 